Title Page

\title{
Improving paediatric advance care planning: results of a learning needs analysis and
} simulation-based education program

Original Article

Naomi T Katz ${ }^{1-4}$, Bronwyn H Sacks ${ }^{1}$, Jenny L Hynson ${ }^{1,3,4}$, Melissa Heywood ${ }^{1,2}$, Molly Williams ${ }^{1}$, Jenni Sokol 2,3

${ }^{1}$ Victorian Paediatric Palliative Care Program, Royal Children's Hospital, Parkville, VIC, Australia

${ }^{2}$ The Royal Children's Hospital Simulation Program, Department of Medical Education

${ }^{3}$ Department of Paediatrics, University of Melbourne, Melbourne, VIC, Australia

${ }^{4}$ Clinical Paediatrics Group, Murdoch Children's Research Group, Melbourne, VIC, Australia

Royal Children's Hospital, 50 Flemington Rd, Parkville 3052

Corresponding author:

Naomi T Katz

Victorian Paediatric Palliative Care Program

Royal Children's Hospital, 50 Flemington Rd, Parkville 3052

Email: naomi.katz@rch.org.au

Phone: +6139345 5374

Acknowledgements:

We thank the children and their families who trust us to care for them during a very difficult time in their lives. We learn from you, and this helps us to continually strive to provide our best care possible. We also thank the Simulation, Palliative Care and Oncology teams for their time and support of this work.

Conflict of interest:

None declared

*Additional workplace addresses for Naomi Katz:

Palliative Care Service, Alfred Health, Melbourne, VIC, Australia

Parkville Integrated Palliative Care Service, The Royal Melbourne Hospital, Melbourne, VIC, Australia

\begin{abstract}
Aim: To assess clinicians' experience, attitudes and confidence with advance care planning (ACP) at a quaternary paediatric referral centre using a learning-needs survey, and then apply this information to develop and examine the feasibility of simulation-based education for this topic.

This is the author manuscript accepted for publication and has undergone full peer review but has not been through the copyediting, typesetting, pagination and proofreading process, which may lead to differences between this version and the Version of Record. Please cite this article as doi: $10.1111 /$ jpc.15074
\end{abstract}

This article is protected by copyright. All rights reserved. 
Methods: An electronic learning-needs survey was distributed to medical, nursing and allied health clinicians from departments who provide primary care for children with life-limiting conditions. Results were incorporated into the design of a simulation-based education session which was piloted with the Royal Children's Hospital Oncology Department.

Results: A total of 157 learning-needs surveys were included in analysis, and included quantitative and qualitative responses from nursing, allied health, senior and junior medical staff from intensive care, general and subspecialty medical teams. Most (75.0\%) participants had participated in ACP discussions previously. Only $40.1 \%$ of participants felt confident to assess appropriate timing of, and $45.2 \%$ felt adequately prepared to participate in ACP discussions. Barriers identified were both clinician and patient/parent related, including clinicians not knowing when to address issues $(43.9 \%)$ or what to say (21.0\%). Participants indicated that ACP discussions are most frequently initiated when death is clearly imminent. Following a pilot of simulation-based education with the oncology department, $90 \%$ of participants expressed confidence to participate in ACP discussions.

Conclusion: Barriers to paediatric ACP, including lack of clinician training, continue to contribute to delayed conversations. Simulation-based education is a valuable and feasible educational tool that can increase clinicians' understanding and confidence in this area.

\section{Key words}

Advance care planning, education, simulation, paediatrics, palliative care

\section{What is already known on this topic;}

1. When completed sensitively and appropriately, advance care planning (ACP) for children with life-limiting conditions benefits clinicians, patients and families,

2. Often a child's deterioration and death can be anticipated,

3. Despite this, ACP tends to happen late in a child's illness course.

\section{What this paper adds}

1. This paper supports clinician appetite for training and education in ACP;

2. This paper also illustrates how simulation-based education is a valuable and feasible tool that can increase clinician understanding and confidence in this area. 


\section{Improving paediatric advance care planning: results of a learning needs analysis and simulation-based education program}

\section{Introduction}

Hundreds of children in Victoria live with and/or die from a life-limiting condition each year. While resuscitation plans are important, advance care planning (ACP) is a much broader concept. $(1,2)$ Advance care planning (ACP) is "a process of discussions between families and health care providers about preferences for care, treatments and goals in the context of the patient's current and anticipated future health".(3) Treating physicians generally lead discussions, however any health professional involved in a child's care may find themselves involved in ACP conversations. ACP allows the treating team to understand what is important to a patient and their family, and share prognostic information and decision-making ahead of potential deterioration. Often deterioration can be anticipated, yet ACP tends to happen late, sometimes in the last days or hours of life. $(2,4,5)$

In Durall et al's study, the top three barriers to paediatric ACP were clinician perception of unrealistic parent expectations, differences between clinician and patient/parent understanding of prognosis, and lack of parent readiness to have the discussion.(4) Clinicians also worry about taking away hope, losing trust of the patient/family, and not knowing the right thing to say.(4) While no parent is ever "ready" for their child to die, many parents consider the possibility that their child might die and the value of life-sustaining treatments prior to physicians initiating ACP.(6) Parents may feel they have little control over their child's last days and wish they had made decisions differently.(6) ACP aims to mitigate regret that may accompany bereavement.

Basu and Swil demonstrated clinician appetite for ACP-focused resources and training.(1) While resources do exist, they are only helpful if clinicians are aware of them. Examples of existing resources include the web and app-based Victorian Government Thinking Ahead resources, the Medical Treatment Planning and Decisions Act 2016 and a UK based framework for medical treatment decision-making for children with life-limiting conditions. $(5,7,8)$ The Thinking Ahead resources provide education, prompts and language to guide ACP conversations at different stages of a child's illness (appendix 1). Similarly, it is vital that clinicians are aware of local systems for documenting and retrieving ACP discussions. This is particularly relevant in the era of transition from paper to electronic medical records (EMR).

Our objectives were to;

(1) build on existing literature by exploring local clinicians' perceptions about the role and barriers to $A C P$, confidence with ACP, and understanding of RCH EMR's capabilities to document and retrieve ACP conversations, and,

(2) design and evaluate a training program that serves operational needs.

\section{Methods}


This work was conducted in partnership with the Palliative Care and Simulation teams at a quaternary paediatric hospital.

Study data were collected and managed using REDCap@ (Research Electronic Data Capture), a secure, web-based software platform that supports data capture and analysis for research studies. $(9,10)$

Our learning-needs survey was partly based on a North American study by Durall et al, in which respondents were asked to rate the frequency of 23 potential barriers.(4) We asked respondents to indicate the top five barriers.

The learning-needs survey was distributed to hospital staff who provide continuing care for children with life-limiting conditions, via departmental and professional distribution lists to reach medical, nursing and allied health staff.

Responses were collected between February $15^{\text {th }}$ and March $17^{\text {th }} 2019$, with one reminder email, and analysed using REDCap (C and Microsoft Excel.

Results were used to develop a practical education session with the following overall learning objectives;

1. Define the role and key principles of ACP discussions in the paediatric setting,

2. Describe the Thinking Ahead ACP framework,

3. Demonstrate where to document and retrieve ACP discussions/resuscitation plans in the Electronic Medical Record (EMR).

A simulation-based education model was chosen as it allows individuals and multidisciplinary teams to practice relatively low-frequency but high-stakes tasks in a safe environment.(11) A professional actor was utilised for their invaluable role in both simulated conversations and feedback. (12)

The scenarios were designed by the Simulation Faculty and reviewed by content experts. Specific scenario objectives aimed to address issues that arose from the needs analysis, in particular the barriers to family discussions, and utilising the ACP communication framework. The scenario content was based on an amalgamation of real cases and included medical and psychosocial details. Actor briefings clarified medical facts, and provided suggested emotions and responses to portray during the simulated scenario as per the Program to Enhance Relational and Communication Skills (PERCS) model.(13)

Sessions were piloted and evaluated with RCH Liquids/Bone Marrow Transplant and Solids Oncology streams. The Simulation Faculty comprised members of the Simulation department, and senior members of the Oncology and Palliative Care departments present as content experts.

Participants were emailed a link to the Thinking Ahead resources as pre-reading. Sessions were designed to be safe, confidential and respectful, opening with the Harvard Basic Assumption ${ }^{\mathrm{TM}}$ that all participants are intelligent, capable, care about doing their best, and want to improve.(14) Acknowledgment was made of the potentially challenging content, with suggested strategies in 
case needed, such as stepping out or approaching Simulation Faculty.

Sessions commenced with an interactive workshop, including review of the Thinking Ahead resources and EMR functionality. This was followed by role-play between participants, and an immersive simulation session where some participants were able to progress conversations with a professional actor, independent of the Simulation Faculty, playing the role of a parent. One case was used for each session, with clinical progression allowing participants to practice different stages of ACP discussions, for example at diagnosis of a possibly incurable disease and subsequently at disease progression despite treatment. Samples of the Liquids/Bone Marrow Transplant scenario are provided in appendix 2 and appendix 3 , with briefs for role-play between participants and role-play with the actor.

The session utilised the framework used in all RCH Simulation communication programs; including a pre- and post-huddle, before and after the 'main' conversation. Sessions were designed to be departmental and inter-professional.

Conversations between the actor and participants were live-streamed to the remaining participants and Simulation Faculty, and were followed by a debrief session which included the professional actor providing feedback about their character's experience. The purpose of the debrief was to reflect on the emotional experience, consider what went well and what might be done differently in the future, and share ideas within the group.

At the conclusion of each session, participants were asked for to complete evaluation forms electronically to provide feedback on the session overall, individual components of the session, key learnings and confidence about each of the learning objectives (appendix 4). Data were collected and analysed using descriptive statistics, Likert scales and qualitative description of free text. Qualitative data from free text were analysed according to grounded theory techniques and qualitative description in order to develop a theoretical framework of participants' perceptions.(15, 16)

Ethics approval was obtained through the hospital's Human Research Ethics Committee (HREC reference: QA/51815/RCHM-2019).

\section{Results}

\section{Results of the learning-needs analysis}

A total of 157 surveys were received and included in data analysis; 142 (90.4\%) were completed in entirety. Table 1 and 2 show respondents' specialties and professions (a number of respondents work across more than one specialty). Paediatric and neonatal intensive care accounted for almost half of the respondents. Eighty-two (52.2\%) participants had worked in their field for more than ten years, and 117 (75.0\%) had previously participated in ACP discussions. 
Figures 1 and 2 illustrate respondents' perceptions about the purposes of and barriers to ACP conversations. Barriers were both clinician and patient/family related. Participants indicated that in their experience, ACP conversations are generally initiated late in a child's illness course (figure 3 ).

Sixty-three (40.1\%) respondents agreed or strongly agreed that they feel confident to assess appropriate timing to initiate, and $71(45.2 \%)$ agreed or strongly agreed that they feel adequately prepared to participate in ACP discussions.

Most respondents were not aware that in addition to functionality enabling documentation of resuscitation status, the local EMR has a dedicated space to document ACP conversations. Of those who were aware of this function, many did not know how it is accessed (figure 4).

\section{Simulation-based education sessions}

Sessions ran for 2.5 hours. While longer sessions would have been preferable, clinical workload was taken into account, particularly given multiple people from the same team were released from clinical work.

Nineteen evaluations were completed ( $100 \%$ response rate). The feedback was overwhelmingly positive; on a 4-point Likert scale the session was rated as "very good" by 18 (95\%) participants, and "good" by 1 (5\%) participant. No participants rated the session as "fair" or "poor". Role-play with a skilled, professional actor, as well as the actor participating in the debrief, was highly valued.

Themes arising from participant reflections included:

1. Consideration of barriers; the benefits of practicing communication techniques and an ACP framework to understand and dissolve barriers to ACP conversations

'Usefulness of a framework to support these conversations.'

'Excellent techniques and sharing communication ideas.'

2. A shared experience; the importance of utilising and sharing expertise and ensuring an interprofessional approach both for families, and also for training of staff

'Learning with clinicians we work with is very valuable, creates a reflective space to unpack one of the most difficult areas of our work together, there is something very powerful about that.'

'Amazing to collaborate with other members of the team. Some great new skills to ensure we are really listening to families and planning.' 
3. Enabling learning; the value of practicing the ACP communication framework in a safe simulated environment with a skilled professional actor to potentially assist in breaking down barriers to facilitating ACP conversations

'Such a valuable experience - very daunting to do the simulation but incredibly helpful and something I can definitely incorporate into practice.'

'Outstanding - education, practical, loved the actor - so skilled.'

Key learnings included the importance of considering the family's agenda, communication techniques, the value of teamwork/utilising the expertise of colleagues, the 'pre-' and 'post-huddle' and Thinking Ahead resources.

Following the session, 17 (90\%) participants agreed or strongly agreed that they felt confident with each of the session's objectives. While two participants strongly disagreed to feeling confident with the objectives, this was not concordant with their overall rating of the session ("very good") and qualitative comments (for example, 'Very relevant, reflective and able to learn').

Participants suggested longer sessions with more scenarios and emotions, more in-depth review of the Thinking Ahead resources, legislation, and incorporation of an adolescent patient for future sessions. Many suggested that sessions should be offered in an ongoing capacity to all teams and include senior medical staff, ideally in a mandatory capacity. Much of this was summarised in the following response:

'Wow - loved the session. Emphasis on doctors not going it alone- try to always tackle with others. Shared experience gives strength and debrief opportunity in these scenarios. Sometimes if alone can be vulnerable to ruminating over how it went/was received. Particularly for medical staff, looking for ongoing opportunities to do joint ACP discussion with other consultants involved e.g. onc, ICU, pall care, is also extremely valuable ongoing learning even for senior consultants. Tackling Adolescent ACP including strategies for talking to a young person would be amazing.'

\section{Discussion}

\section{Barriers to ACP: describing and addressing barriers to ACP conversations}

Our findings suggest that barriers to paediatric ACP continue to be a real issue for clinicians. This is despite recognition that families and clinicians benefit from early ACP, and that many parents have thought about these issues prior to clinicians initiating conversations. $(1,4-6)$ Like Durall et al, unrealistic parent expectations and differences between clinician and patient/parent understanding of prognosis were the top barriers to ACP in our study.(4) We want to highlight that clinician perception of parental expectation, and actual parental expectation, may differ. Awareness and separation between clinician and family experiences and needs is important to ensure that families are at the centre of decision-making. This is further highlighted by findings that other clinician- 
related factors, such as concern about taking away hope and unrealistic clinician expectations, were commonly reported barriers.

Some factors and barriers are unique to paediatric ACP, including parents acting as substitute decisionmakers.(17) In addition, while advance care planning conversations for children are used to guide future care, they are not legally binding.(5) This is distinct from advance care planning in adults which provides adults with decision-making capacity an opportunity to draw up a legally binding document that outlines their wishes and preferences for future treatment in the event that they lose decisionmaking capacity.(18) Processes and terminology vary between states; for example, in Victoria this legally-binding document is called an Advance Care Directive. (19) While ACP is not legally binding in paediatrics, there are ethical imperatives to have these conversations as they stand to benefit the child, as well as their parents and treating clinicians. For example, ACP may limit a child's exposure to burdensome and unhelpful interventions and mitigate parental regret in bereavement by empowering them to make decisions using honest and accurate prognostic information.

Results of the learning-needs survey, including the roles and barriers to ACP, were discussed in the workshop. This provided participants a reflective space to consider and discuss their own experiences and perceptions, and then go on to practice the conversations. For example, discussing and practicing the Thinking Ahead framework with suggested ACP triggers and language was used to help address the third most significant barrier reported by our cohort that clinicians do not know the right time to initiate ACP discussions. Simulation-based education was utilised as a tool to reinforce knowledge and train participants to utilise the ACP framework when having these difficult discussions with families. The simulation session was designed to provide opportunity to utilise the ACP framework during discussion with a family, and then discuss the barriers to discussion in the debrief along with the challenges in delivering the framework, with the aim to encourage participants to discover their gaps in knowledge and come up with solutions to these issues.

The relative high concern about clinicians not knowing the right time to address ACP compared with Durall et al's study may be related to differences in survey audiences; Durall et al only included oncology and intensive care clinicians (4) compared with our more heterogenous audience in which just over half the respondents were from intensive care and oncology. The relatively high representation of intensive care clinicians in our study likely reflects higher staff numbers and prevalence of deaths. Differences in survey findings highlight the value of replicating learning-needs surveys in different settings to interrogate local experience and tailor findings and interventions accordingly.

Our findings support the literature that ACP conversations occur late in a child's illness course. $(1,4)$ The 2016 'National consensus statement: essential elements for safe and high-quality paediatric end-of-of-life care' highlights that systems to support ACP discussions align with Australian Commission on National Safety and Quality in Health Care Standards. $(20,21)$ Sensitive and appropriately-timed ACP discussions should be core business for all healthcare institutions.

Excellent resources to guide clinicians about conducting, documenting and retrieving ACP exist, however these are only useful if clinicians are aware of their existence. Communication skills training programs are most effective when they are learner-centred, and focus on practicing skills with the 
best strategies including role-play, feedback and small-group discussions.(22) Our overwhelmingly positive feedback supports simulation-based training as a powerful method to upskill in the communication-based procedure of ACP. Despite simulation potentially being confronting, it was positively accepted. Starting with role-play eased participants into the environment, prior to the potentially more daunting experience of being observed by colleagues with an actor, and allowed all participants the opportunity to practice.

\section{Simulation-based education for ACP: enabling learning through shared experience}

While resuscitation discussions and documentation are the responsibility of medical staff, broader advance care planning conversations are usually interprofessional at our institution and medical, nursing and allied health clinicians are all encouraged to document and retrieve elements of ACP discussions in the EMR. Hence, we deliberately included medical, nursing and allied health clinicians in both our learning-needs survey and workshops. An interprofessional departmental session ideally provides a safe space to learn and share vulnerability with colleagues, reinforcing the value of different expertise and skillsets, and the complementary roles of medical, nursing and allied health staff in ACP conversations.

Our interprofessional departmental sessions afforded participants an opportunity to practice conducting a 'pre-huddle' to share and align their thoughts rather than potentially coming across as fragmented, disorganised and confusing to a family. Participants were encouraged to consider who should be present in the conversation with the family, e.g doctor +/- clinical nurse coordinator +/social worker, thinking about the needs of the family in terms of expertise and numbers of clinicians present. In our sessions, we utilised the Thinking Ahead resources for the ACP conversation.

Participants discussed the benefits of a 'post-huddle', including the opportunity for clinicians to review the conversation, plan follow up, debrief as needed, and document appropriately.

There may be a role for both intra- and inter-departmental simulation sessions to foster clinician relationships within and between teams. The heterogeneity of respondents to our learning-needs survey allows results to be analysed and presented according to specialty and profession. These results could be used to tailor scenarios and education sessions to departments, professions and institutions. For example, our study highlights how workshops may be tailored to local or systemic issues, for example difficulties in ACP documentation and retrieval that may be arise during transition from paper to electronic medical records.

\section{Strengths and limitations of the study}

The study was conducted at a single hospital site, and simulation sessions were only conducted with a single hospital department, potentially limiting generalisability. However, the heterogeneity of survey respondents is a strength, and potentially helpful as results can be used to target particular audiences. Self-selection bias for the learning-needs survey needs to be considered given it was optional. Although only two workshops were conducted, participant feedback was positive and useful for future sessions. Given that the same two people consistently selected items at the end of the Likert scale that did not correspond to their overall session rating, this was possibly a result of 
innate difficulties with surveys, such as misreading of the position of Likert scale items, and needs to be considered in designing future evaluations. Pre-reading before sessions potentially saves time, however usefulness is limited if not read in advance. We did not specifically ask participants in the evaluation whether they had read Thinking Ahead resources provided as pre-reading, however this question would have been useful to allow comparison of post-workshop evaluation responses based on whether pre-reading was done and will be included in the future. Finally, given five of the six authors work in the Department of Palliative Care, we recognise the possibility of 'insider status' creating an unconscious bias and may hence have limited the development and collation of evaluation results.

\section{Conclusions}

When completed sensitively and appropriately, ACP benefits clinicians, patients and families. Our experience supports existing literature about the barriers to ACP, and recognition of the need for further training. However, it goes a step further to illustrate how simulation-based education is a valuable and feasible tool that can increase clinician understanding and confidence in this area. Our study illustrates clinician appetite for simulation-based ACP training.

\section{References}

1. Basu S, Swil K. Paediatric advance care planning: Physician experience and education in initiating difficult discussions. J Paediatr Child Health. 2018;54:510-4.

2. Tsai E. Advance care planning for paediatric patients. Paediatr Child Health. 2008;13(9):791 - 6.

3. Spicer S, Macdonald ME, Davies D, Vadeboncoeur C, Siden H. Introducing a lexicon of terms for paediatric palliative care. Paediatr Child Health. 2015;20(3):155-6.

4. Durall A, Zurakowski D, Wolfe J. Barriers to Conducting Advance Care Discussions for Children With Life-Threatening Conditions. Pediatrics. 2012;129(4):e975-e82.

5. Victorian Government. Thinking ahead framework: Planning care for children with life-limiting conditions2016. Available from: www.health.vic.gov.au/acp.

6. Meyer EC, Burns JP, Griffith JL, Truog RD. Parental perspectives on end-of-life care in the pediatric intensive care unit. Pediatric Critical Care. 2002;30(1):226-31.

7. Medical Treatment Planning and Decisions Act, (2016).

8. Larcher V, Craig F, Bhogal K, Wilkinson D, Brierley J. Making decisions to limit treatment in life-limiting and life-threatening conditions in children: a framework for practice. Arch Dis Child. 2015;100:s1 - s23.

9. Harris PA, Taylor R, Minor BL, Elliott V, Fernandez M, O'Neal L, et al. The REDCap consortium: Building an international community of software partners. J Biomed Inform. 2019. 
10. Harris PA, Taylor R, Thielke R, Payne J, Gonzalez N, Conde JG. A metadata-driven methodology and workflow process for providing translational research informatics support. J Biomed Inform. 2009;42(2):377 - 81.

11. Burke CS, Salas E, Wilson-Donnelly K, Priest H. How to turn a team of experts into an expert medical team: guidance from the aviation and military communities. Quality \& Safety in Health Care. 2004;13(Suppl 1):i96-104.

12. Bell SK, Pascucci RC, Fancy KM, Coleman K, Zurakowski D, Meyer EC. The educational value of improvisational actors to teach communication and relational skills: Perspectives of interprofessional learners, faculty, and actors. Patient Educ Couns. 2014;96:381-8.

13. Browning DM, Meyer EC, Truog RD, Solomon MZ. Difficult Conversations in Health Care: Cultivating Relational Learning to Address the Hidden Curriculum Academic Medicine: Journal of the Association of American Medical Colleges. 2007;82(9):905-13.

14. Center for Medical Simulation. The Basic Assumption(TM) Boston [Available from: https://harvardmedsim.org/resources/the-basic-assumption/.

15. Sandelowski M. Whatever happended to qualitative description? Res Nurs Health. 2000;23(4):334-40.

16. Charmaz K. Construcing Grounded Theory. A Practical Guide Through Qualitative Analysis. London: Sage Publications; 2006.

17. Lotz JD, Jox RJ, Borasio GD, Fuhrer M. Pediatric advance care planning from the perspective of health care professionals: A qualitative interview study. Palliat Med.

2015;29(3):212-22.

18. Austin Health. Advance Care Planning Australia 2018 [Available from:

https://www.advancecareplanning.org.au/resources/advance-care-planning-for-your-stateterritory.

19. Medical Treatment Planning and Decisions Act, (2016).

20. Australian Commission on Safety and Quality in Health Care. National Consensus Statement: Essential elements for safe and high-quality paediatric end-of-life care. Sydney: ACSQHC; 2016.

21. Australian Commission on Safety and Quality in Health Care. National Safety and Quality Health Service Standards. Sydney; 2017.

22. Berkhof M, van Rijssen HJ, Schellart AJM, Anema JR, van der Beek AJ. Effective training strategies for teaching communication skills to physicians: An overview of systematic reviews. Patient Educ Couns. 2011;84(2):152-62.

This article is protected by copyright. All rights reserved. 
Table 1. Specialty of respondents to learning-needs survey

\begin{tabular}{|l|r|}
\hline & $\mathrm{n}(\%)$ \\
\hline Paediatric intensive care & $46(29.3)$ \\
\hline Neonatal intensive care & $28(17.8)$ \\
\hline Oncology & $19(12.1)$ \\
\hline Neurodevelopment and disability & $15(9.6)$ \\
\hline General paediatrics & $14(8.9)$ \\
\hline Respiratory & $12(7.6)$ \\
\hline Palliative care & $10(6.4)$ \\
\hline Gastroenterology & $6(3.8)$ \\
\hline Cardiology & $5(3.2)$ \\
\hline Neurology & $4(2.5)$ \\
\hline Nephrology & $3(1.9)$ \\
\hline Metabolics & $1(0.6)$ \\
\hline
\end{tabular}

Table 2. Profession of respondents to learning-needs survey

\begin{tabular}{|l|r|}
\hline & \multicolumn{1}{|c|}{ (\%) } \\
\hline Nursing staff & $77(49.0)$ \\
\hline Senior medical staff & $31(19.7)$ \\
\hline Social worker & $18(11.5)$ \\
\hline Junior medical staff & $14(8.9)$ \\
\hline Physiotherapist & $6(3.8)$ \\
\hline Occupational therapist & $6(3.8)$ \\
\hline Missing & $2(1.9)$ \\
\hline Chaplain & $1(0.6)$ \\
\hline Dietitian & $1(0.6)$ \\
\hline
\end{tabular}

Figure 1. Respondent, $n(\%)$, perception of the purpose(s) of ACP (more than one response possible) 


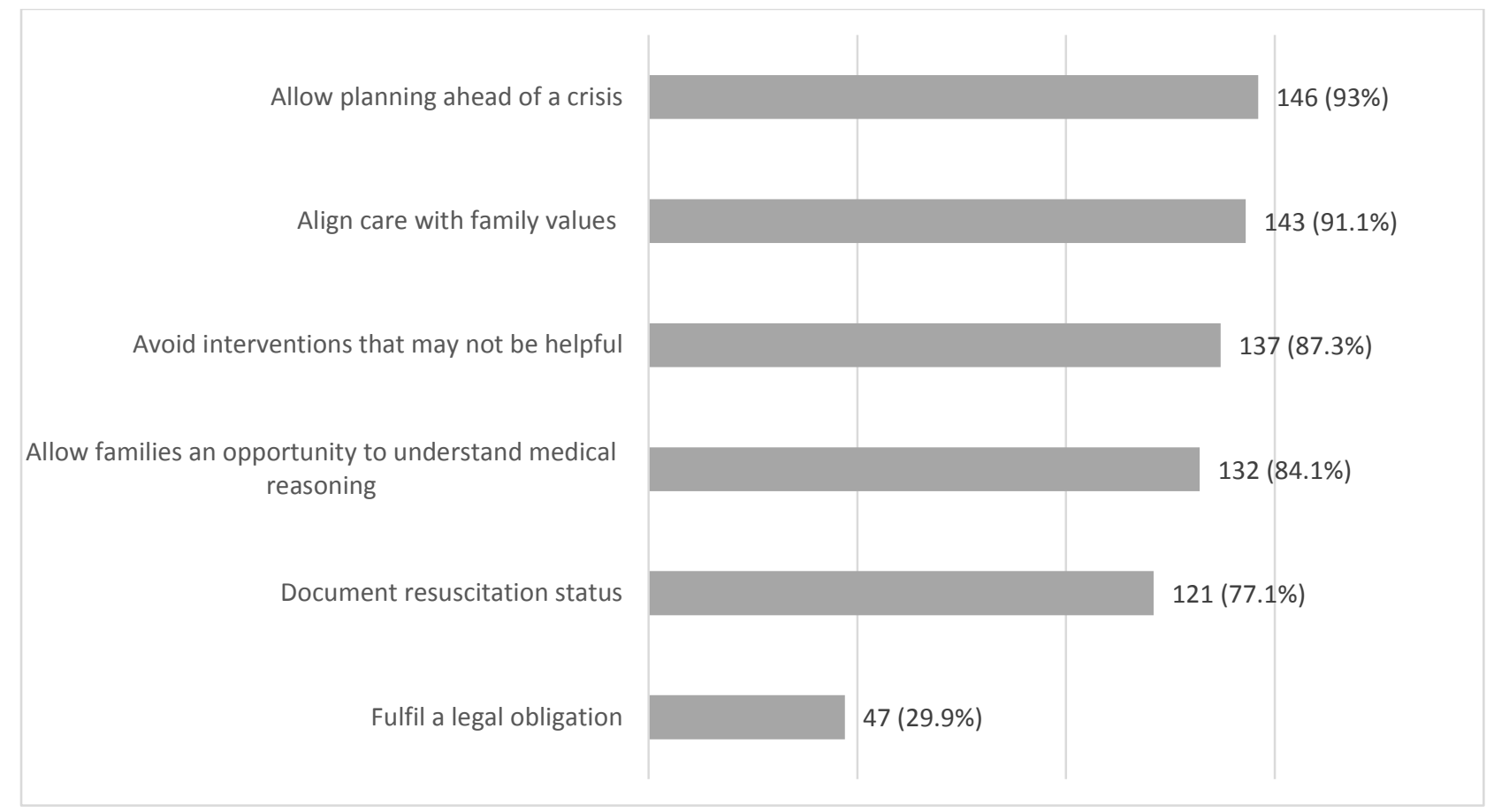

Figure 2. Perceived barriers to ACP: proportion of respondents, $n(\%)$, who rated each barrier among the top five barriers to ACP 
Differences between clinician and patient/parent understanding of prognosis

Lack of parent readiness to have the discussion

Clinicians not knowing the right time to address the issues

Clinician uncertainty about prognosis

Unrealistic parent expectations

Clinician concern about taking away hope

Inability of parent to fully comprehend the issues

Ethical considerations

Clinicians not knowing the right thing to say

Unrealistic clinician expectations

Lack of clinician relationship with patient/family

Concern about losing the trust of the parent

Lack of clinician time

Conflict between parent and other family members

Lack of patient readiness to have the discussion

Lack of importance to clinicians

Concern about losing the trust of the patient

Conflict between patient and parent

Concern that the patient would receive less attention from clinicians

Unrealistic patient expectations

Inability of school-aged patients to fully comprehend the issues
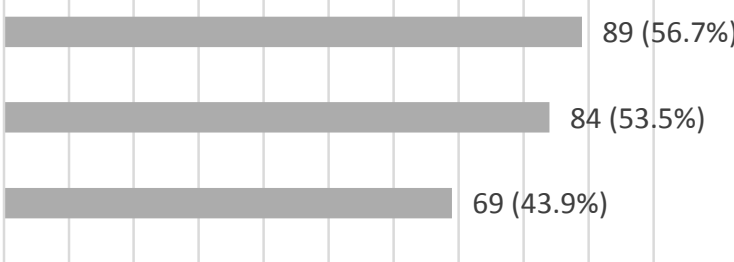

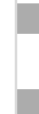

$60(38.2 \%)$
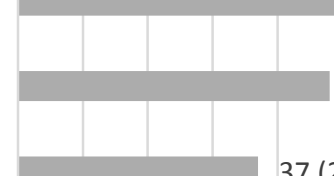

$48(30.6 \%)$

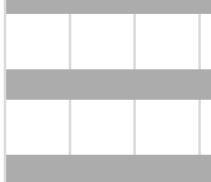

$37(23.6 \%)$

$34(21.7 \%)$

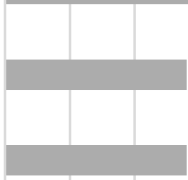

$33(21.0 \%)$

$28(17.8 \%)$

$28(17.8 \%)$

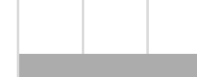

$28(17.8 \%)$
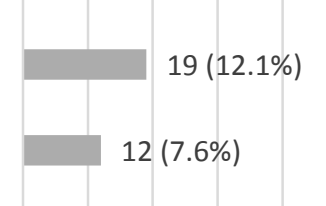

$10(6.4 \%)$

$10(6.4 \%)$

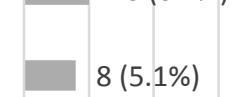

$5(3.2 \%)$

$5(3.2 \%)$

$2(1.3 \%)$

.

$2(1.3 \%)$

Inability of adolescent patients to fully comprehend the issues

$2(1.3 \%)$

Laws and regulations

$1(0.6 \%)$

$\begin{array}{lllllllllll}0 & 10 & 20 & 30 & 40 & 50 & 60 & 70 & 80 & 90 & 100\end{array}$ 
Figure 3. Respondent experience, $n(\%)$, of when ACP conversations are initiated (more than one option allowed)

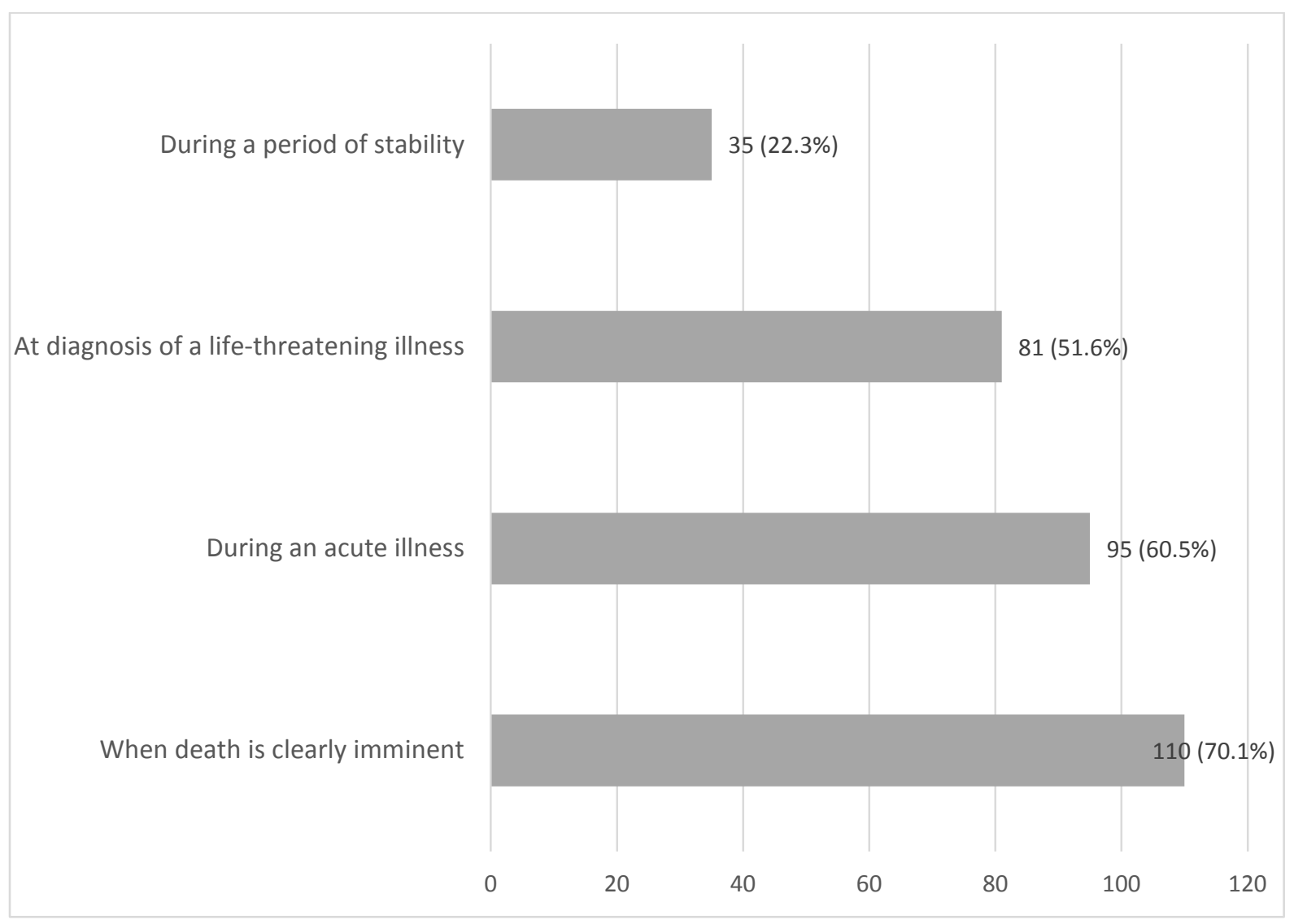

Figure 4. Knowledge about the EMR function to document and retrieve ACP conversations

This article is protected by copyright. All rights reserved. 


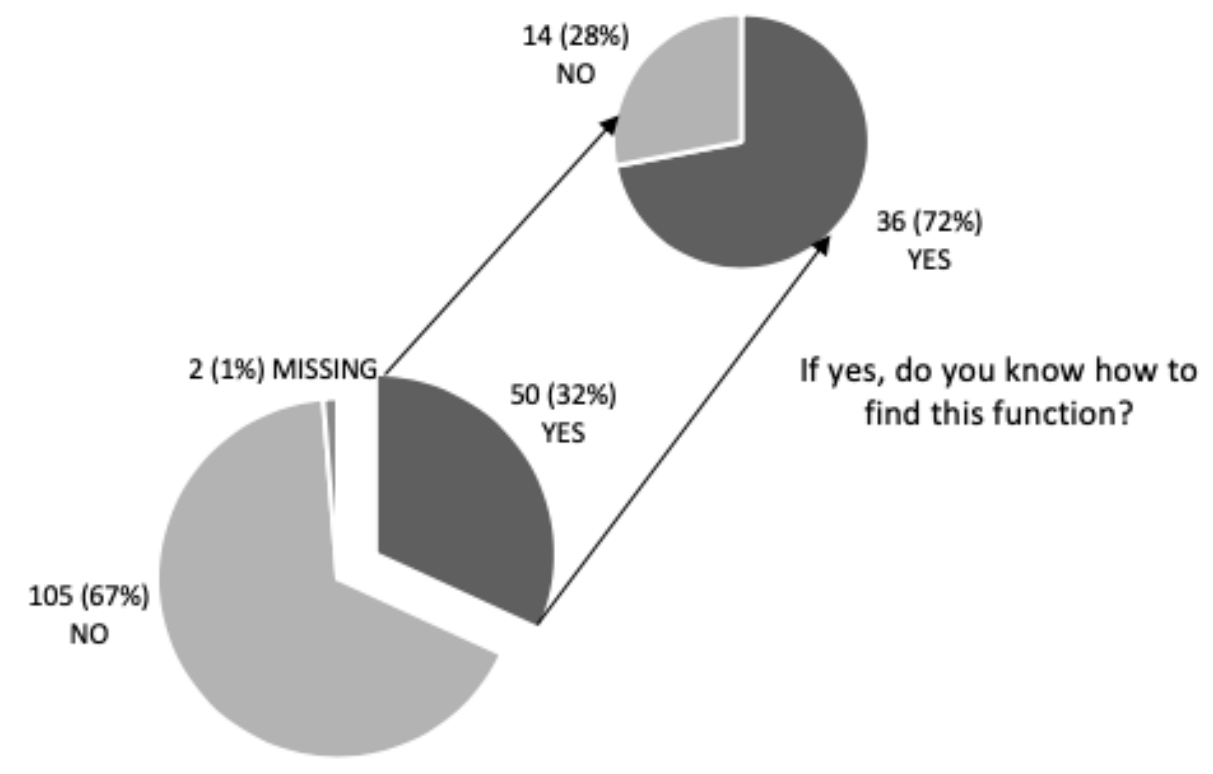

Were you aware of the EMR function to document ACP conversations?

This article is protected by copyright. All rights reserved. 
Title Page

Improving paediatric advance care planning: results of a learning needs analysis and simulation-based education program

Original Article

Naomi T Katz ${ }^{1-4}$, Bronwyn H Sacks ${ }^{1}$, Jenny L Hynson ${ }^{1,3,4}$, Melissa Heywood ${ }^{1,2}$, Molly Williams ${ }^{1}$, Jenni Sokol 2,3

${ }^{1}$ Victorian Paediatric Palliative Care Program, Royal Children's Hospital, Parkville, VIC, Australia

${ }^{2}$ The Royal Children's Hospital Simulation Program, Department of Medical Education

${ }^{3}$ Department of Paediatrics, University of Melbourne, Melbourne, VIC, Australia

${ }^{4}$ Clinical Paediatrics Group, Murdoch Children's Research Group, Melbourne, VIC, Australia

Royal Children's Hospital, 50 Flemington Rd, Parkville 3052

Corresponding author:

Naomi T Katz

Victorian Paediatric Palliative Care Program

Royal Children's Hospital, 50 Flemington Rd, Parkville 3052

Email: naomi.katz@rch.org.au

Phone: +6139345 5374

Acknowledgements:

We thank the children and their families who trust us to care for them during a very difficult time in their lives. We learn from you, and this helps us to continually strive to provide our best care possible. We also thank the Simulation, Palliative Care and Oncology teams for their time and support of this work.

Conflict of interest:

None declared

*Additional workplace addresses for Naomi Katz:

Palliative Care Service, Alfred Health, Melbourne, VIC, Australia

Parkville Integrated Palliative Care Service, The Royal Melbourne Hospital, Melbourne, VIC, Australia 


\section{University Library}

\section{- M M N E R VA A gateway to Melbourne's research publications}

Minerva Access is the Institutional Repository of The University of Melbourne

Author/s:

Katz, NT;Sacks, BH;Hynson, JL;Heywood, M;Williams, M;Sokol, J

Title:

Improving paediatric advance care planning: Results of a learning needs analysis and simulation-based education programme

Date:

2020-08-19

Citation:

Katz, N. T., Sacks, B. H., Hynson, J. L., Heywood, M., Williams, M. \& Sokol, J. (2020). Improving paediatric advance care planning: Results of a learning needs analysis and simulation-based education programme. JOURNAL OF PAEDIATRICS AND CHILD HEALTH, 56 (12), pp.1898-1905. https://doi.org/10.1111/jpc.15074.

Persistent Link:

http://hdl.handle.net/11343/276168 